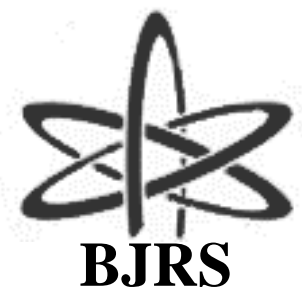
BRAZILIAN JOURNAL
$\mathrm{OF}$
RADIATION SCIENCES
09-01 (2021) 01-21

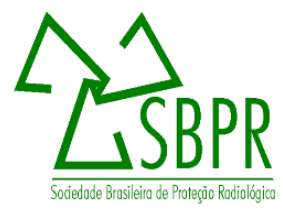

\title{
Modelagem computacional da dispersão atmosférica aplicada a um reator modular de pequeno porte
}

\author{
Curzio $^{a}$ R. C., Neto ${ }^{a}$ A. T., Moura ${ }^{a}$ B. S., Talon ${ }^{a}$ J. D., Lopes ${ }^{a}$ T. J., Bonfim ${ }^{b}$ C. E. S., \\ Cardoso $^{a}$ D. O., Morales ${ }^{\mathrm{a}}$ R. K., Gomes ${ }^{\mathrm{a}}$ R. G., Gavazza ${ }^{\mathrm{a}}$ S. \\ ${ }^{a}$ Instituto Militar de Engenharia (IME) / Seção de Engenharia Nuclear (SE/07). Praça Gen. Tibúrcio, 80, 22290-270, \\ Urca, Rio de Janeiro, RJ, Brasil \\ ${ }^{b}$ Instituto de Defesa Química, Biológica, Radiológica e Nuclear (IDQBRN). Avenida das Américas, 28.705,Guaratiba, \\ 23020-470, Rio de Janeiro, RJ, Brasil \\ rodrigoc.curzio@gmail.com
}

\section{RESUMO}

Este trabalho descreve a modelagem computacional de dispersão atmosférica decorrente de acidente radiológico hipotético em reator modular de pequeno porte (SMR), cuja potência é de 16 MWe (50 MWt). Utilizou-se o software SCALE para modelar o núcleo com três regiões de enriquecimento do combustível, a 4\%, 5\% e 20\%, e obter atividades dos radionuclídeos oriundos de reações nucleares durante o burnup, após 2 anos de operação. Foi escolhida uma localidade de interior para instalação do SMR, onde informações sobre condições meteorológicas foram coletadas para identificação da classe de estabilidade atmosférica predominante. Dentre os radionuclídeos do inventário, considerou-se a contribuição do Cs-137 para simulação, usando-se o código HotSpot, da concentração e das doses totais efetivas (TEDE) recebidas, ambas em função da distância do evento. Os resultados sugerem que a TEDE máxima calculada foi de 3,6 Sv, a $34 \mathrm{~m}$ do reator, diminuindo com o tempo e distância, e seguindo o modelo Gaussiano de dispersão, e que a pluma de contaminação é dependente dos critérios de Pasquill-Gifford e da atividade do Cs-137. Para doses entre $1 \mathrm{mSv}$ e $10 \mathrm{mSv}$ e entre $10 \mathrm{mSv}$ e $50 \mathrm{mSv}$, sugere-se a abrigagem da população nas construções existentes na localidade, e para valores acima de $50 \mathrm{mSv}$, a abrigagem nessas condições ou a evacuação do pessoal das proximidades do reator em movimento contrário ao de propagação da pluma. A relevância dessa investigação mostra a importância do planejamento de respostas em situação de emergência e a influência das condições meteorológicas, considerando-se os dados assumidos na 
simulação.

Palavras-chave: SMR, Modelagem, Dispersão, SCALE, HotSpot.

\begin{abstract}
This paper describes the computational modeling of atmospheric dispersion resulting from a hypothetical radiological accident in the small modular reactor (SMR), with a power of $16 \mathrm{MWe}(50 \mathrm{MWt})$. The software SCALE was used to model the core with three fuel enrichment regions, $4 \%, 5 \%$ and $20 \%$, and to obtain activities of radionuclides from nuclear reactions during burnup, after 2 years of operation. A country town was chosen to install the SMR, where information about meteorological conditions was collected to identify the predominant atmospheric stability class. Among radionuclides in the inventory, the contribution of Cs-137 was considered for simulation, using the HotSpot code, of the concentration and the Total Effective Dose Equivalent (TEDE) received, both depending on the distance from event. The results suggest that the maximum calculated TEDE was $3.6 \mathrm{~Sv}, 34 \mathrm{~m}$ from the reactor, decreasing with time and distance, and following the Gaussian dispersion model, and that the contamination plume is dependent on the Pasquill-Gifford criteria and Cs-137 activity. For doses between $1 \mathrm{mSv}$ and $10 \mathrm{mSv}$ and between $10 \mathrm{mSv}$ and $50 \mathrm{mSv}$, it is suggested that the population be sheltered in the existing buildings in the locality, and for values above $50 \mathrm{mSv}$, the shelter in these conditions or the evacuation of population from the vicinity of the reactor in movement contrary to the spread of the plume. The relevance of this investigation shows the importance of emergency response planning and the influence of weather conditions, considering the data assumed in the simulation.
\end{abstract}

Keywords: SMR, Modeling, Dispersion, SCALE, HotSpot. 


\section{INTRODUÇÃO}

O estudo apresenta a modelagem computacional da dispersão atmosférica decorrente de um acidente radiológico hipotético, em um reator modular de pequeno porte (SMR), do tipo PWR, cuja potência é de $16 \mathrm{MWe}(50 \mathrm{MWt})$. Este reator é projetado para fornecer energia elétrica [1]. Por ser fabricado em módulos, sua versatilidade propicia o transporte e a instalação em locais menos propensos à construção de um reator nuclear convencional, podendo ser uma solução para localidades onde o abastecimento de energia ou o acesso sejam difíceis, como na região Norte do Brasil [2].

Por hipótese, e dentro desse contexto, torna-se relevante uma análise de situação para estudo, por modelagem computacional, buscando-se avaliar a implementação das medidas de proteção em situações de emergência, propostas para evitar ou reduzir a exposição da população à radiação, caso ocorresse um acidente com um reator desse porte, instalado em uma área longínqua. Essa análise permitiria verificar com mais detalhes a seleção adequada do local para a execução de atividades e práticas nucleares com esse tipo de reator modular [1,3].

O objetivo da pesquisa visa realizar a simulação computacional da dispersão atmosférica de radionuclídeos, por meio do código HotSpot Health Physics 3.1.2 [4], liberados durante um acidente hipotético no SMR, cujo núcleo foi modelado com o software SCALE [5], e analisar os dados obtidos, a fim de se verificar a adoção das medidas protetoras imediatas na fase inicial deste evento radiológico postulado, bem como a influência das condições meteorológicas na pluma de contaminação.

\section{MATERIAIS E MÉTODOS}

\subsection{Modelagem do reator SMR}

Para este estudo, assumiu-se que o SMR, cuja potência é de $16 \mathrm{MWe}$ (50 MWt), esteve funcionando continuamente por 2 anos $(720 \text { dias })^{1}$, em regime de potência máxima [6], e que a dispersão atmosférica de radionuclídeos foi gerada pela queima de combustível desse reator. Foram

\footnotetext{
${ }^{1}$ A vida útil do combustível do reator SMR em estudo é estimada em 2 anos [1].
} 
definidos o seu núcleo, conforme a Figura $1^{2}$, com três regiões de enriquecimento de combustível, a $4 \%, 5 \%$ e $20 \%$, bem como o cálculo de inventário de radionuclídeos e, para isso, utilizou-se o software SCALE (KENO VI e T6-DEPL) [5], a fim de modelar a geometria e de obter as atividades médias decorrentes dos radionuclídeos provenientes das reações nucleares ocorridas durante o burnup do combustível, após 2 anos de operação ininterruptos.

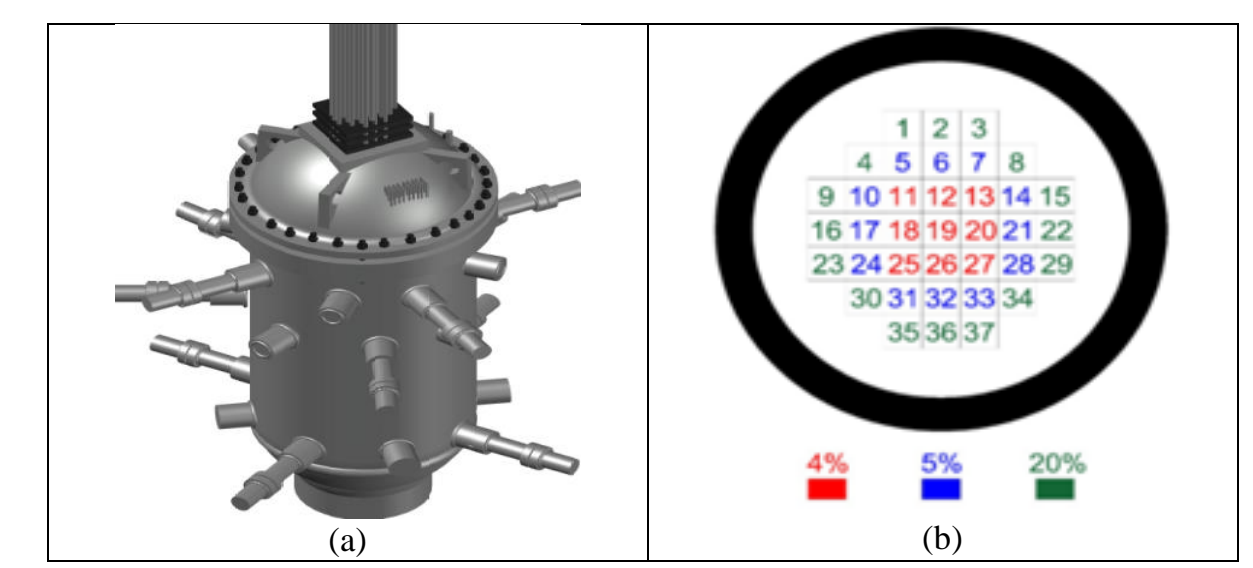

Figura 1: Ilustração da instalação nuclear: a) à esquerda, o vaso de pressão do SMR ; $b$ ) à direita, a vista superior do núcleo.

Fonte: AUTOCAD, 2019 (Figura 1.a); SCALE, 2020 (Figura 1.b)

\subsection{Modelagem da simulação do acidente radiológico}

Para a ocorrrência do acidente radiológico no SMR, foram consideradas as premissas a seguir: a) o reator estava operando na potência máxima, por 2 anos ininterruptos; b) o revestimento (cladding), à base de Zircaloy-4, atingiu valores de temperatura além das suas especificações de projeto; c) o limite de $340^{\circ} \mathrm{C}$ [7] levou à criação de cavidades. Essa elevação de temperatura foi motivada por uma suposta falha nas travas das barras de controle (absorvedores de nêutrons à base de cádmio) e no retardo na inserção de água borada, o que ocasionou, não somente a inserção de reatividade positiva no núcleo, isto é, o reator na condição supercrítica, mas também uma perda substancial de fluido, caracterizando o LOCA (perda acidental de refrigerante). Assim, o desgaste do revestimento interno do reator ocorreu por conta da redução no fluxo de fluido responsável por

\footnotetext{
${ }^{2}$ Ilustra apenas o vaso do reator. Foram desconsiderados todos os componentes e demais acessórios da instalação nuclear.
} 
refrigerar o núcleo, devido ao diâmetro nominal reduzido (inerente à geometria de pequeno porte do SMR) das tubulações do sistema de arrefecimento forçado [8].

Com esse cenário de falha no cladding, foi assumido que os produtos de fissão gasosos foram liberados na água do vaso de pressão do reator e coletados em seu topo, continuando a funcionar sem qualquer substituição dos elementos em condição de falha. Consequentemente, os radionuclídeos em questão foram dispersos, de modo instantâneo, para a atmosfera, por um périodo de 10 min, em uma única liberação. Essa liberação ocorreu através do sistema de venting existente no prédio do reator, localizado a $10 \mathrm{~m}$ acima do solo, conforme descrito pela Figura 2.

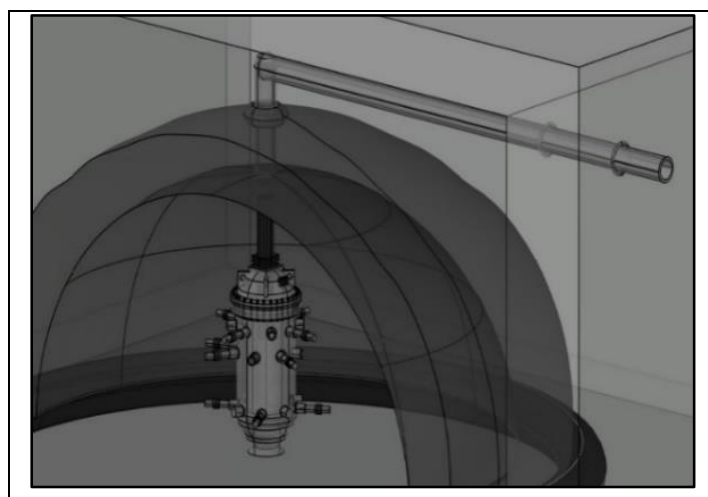

(a)

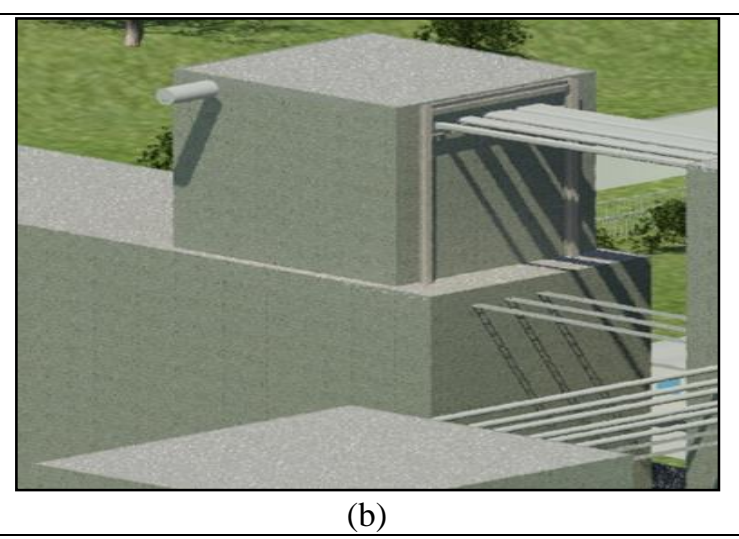

(b)

Figura 2: Ilustração simplificada (fora de escala): a) à esquerda, da contenção para o SMR; $b$ ) à direita, do sistema de venting da instalação nuclear.

Fonte: AUTOCAD, 2019 (Firgura 2.a); REVIT, 2019 (Figura 2.b)

\subsection{Modelagem da dispersão e cálculo de doses}

A partir dos resultados dos radioisótopos obtidos por meio do programa SCALE, e considerando o momento do acidente postulado, a dispersão atmosférica foi calculada mediante o modelo Gaussiano de dispersão de plumas, através do código HotSpot Health Physics 3.1.2 [4].

O HotSpot é um software que leva em consideração a velocidade do vento para o cálculo do transporte do material radioativo liberado no ambiente e a estabilidade atmosférica local, nominadas de A (extremamente instável) a F (moderadamente estável), segundo as classes de estabilidade de Pasquill-Gifford [4]. O programa permite determinar a concentração atmosférica dos agentes radiológicos dispersos em qualquer ponto do espaço, onde a origem do sistema de coordenadas está 
localizada ao nível do solo $(x=0, y=0, z=0)$, com as coordenadas de liberação dos radionuclídeos em $(x=H, y=0, z=0)$, por meio da seguinte equação [4]:

$$
C(x, y, z, H)=\frac{Q}{2 \pi \sigma_{y} \sigma_{z} u} e^{\left[-\frac{1}{2}\left(\frac{y}{\sigma_{y}}\right)^{2}\right]}\left\{e^{\left[-\frac{1}{2}\left(\frac{z-H}{\sigma_{z}}\right)^{2}\right]}+e^{\left[-\frac{1}{2}\left(\frac{z+H}{\sigma_{z}}\right)^{2}\right]}\right\} e^{\left[-\frac{\lambda x}{u}\right]} D F(x)
$$

onde, $C$, consiste na concentração atmosférica $\left(B q . s / m^{3}\right) ; Q$, na atividade da fonte $(B q) ; \sigma_{y}$, do desvio padrão da distribuição de concentração na direção perpendicular à do vento $(m) ; \sigma_{z}$, do desvio padrão da distribuição de concentração na direção vertical $(m) ; y$, na distância perpendicular à direção do vento $(m) ; z$, na distância do eixo vertical $(m) ; H$, na altura efetiva ${ }^{3}$ de lançamento $(m) ; \lambda$, na constante de decaimento radioativo $\left(s^{-1}\right) ; x$, na distância percorrida na direção do vento $(m) ; u$, na velocidade média do vento na altura de liberação efetiva do material $(\mathrm{m} / \mathrm{s}) \mathrm{e} ; D F(x)$, no fator de depleção da pluma, calculado pela seguinte equação [4]:

$$
D F(x)=\left\{\exp \int_{0}^{x} \frac{1}{\sigma_{Z}(x) \exp \left[\frac{1}{2}\left(\frac{H}{\sigma_{Z}(x)}\right)^{2}\right]} d x\right\}^{-\frac{v}{u} \sqrt{\frac{2}{\pi}}}
$$

onde $v$ é a velocidade de deposição $(\mathrm{m} / \mathrm{s})$ do material radioativo, e as demais variáveis estão de acordo com a equação (1). A Figura 3 ilustra esquematicamente o processo de dispersão de radionuclídeos com base no modelo gaussiano.

Para a simulação da dose total efetiva $\left(\mathrm{TEDE}^{4}\right)$, o HotSpot usa as metodologias de dosimetria de radiação recomendadas pela International Commission on Radiological Protection (ICRP), para converter a concentração fornecida pela equação (1) na dose a que serão expostos os indivíduos que permanecerem no mesmo local e na direção do vento. Para isso, o código utiliza os valores de coeficientes de dose a partir dos documentos Federal Guidance Report (FGR) 11, 12 e 13, que fornecem os fatores de conversão de dose por inalação, submersão e ingestão [3,4].

\footnotetext{
${ }^{3}$ Para este trabalho, assumiu-se, por hipótese, que a altura efetiva de lançamento é a própria altura física de liberação do material radioativo para a atmosfera (neste caso, a própria altura do sistema de venting, de $10 \mathrm{~m}$ em relação ao solo) [4]. ${ }^{4}$ A TEDE (Total Effective Dose Equivalent) é a soma entre a dose efetiva para exposições externas (submersão, ressuspensão e deposição) e a dose absorvida comprometida para exposições internas (inalação) [10].
} 


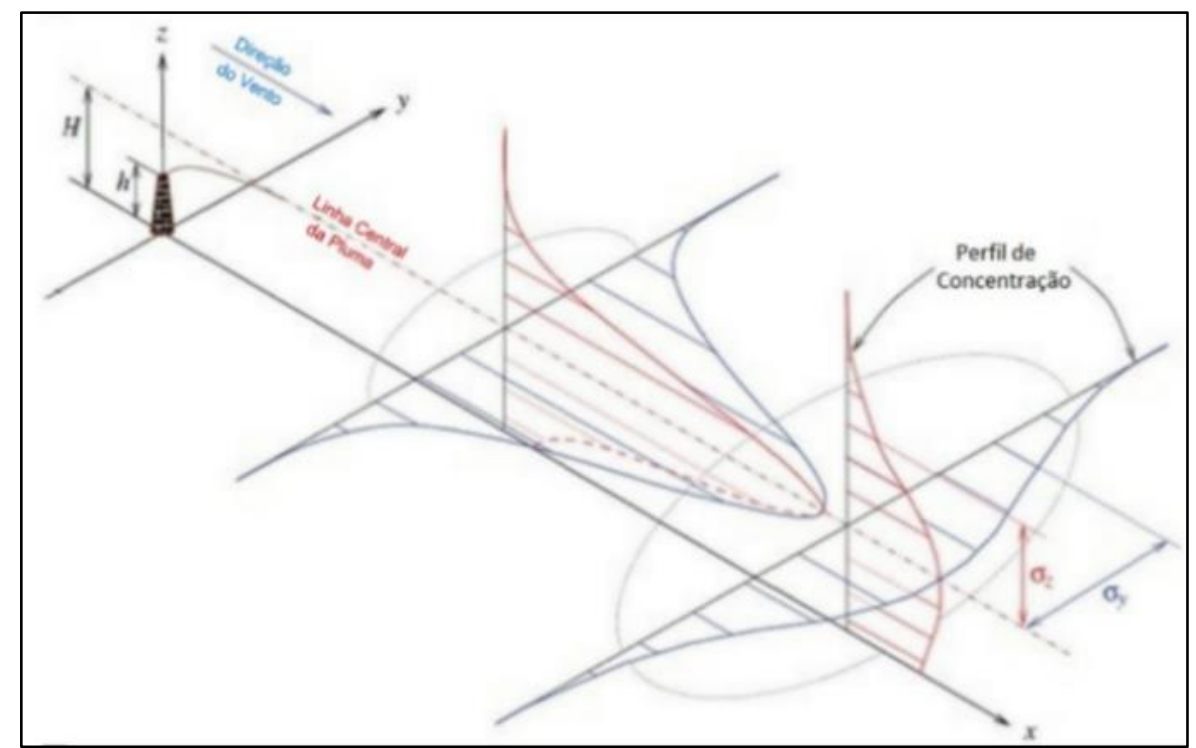

Figura 3: Representação ilustrativa da dispersão de plumas radiológicas, a partir de uma fonte pontual contínua, com a direção do vento alinhada com o eixo $x$. Os perfis de concentração são dados na direção vertical, em vermelho, e na horizontal, em azul.

Fonte: Adaptado de STOKIE, 2011 [9]

A área escolhida para as simulações está localizada no interior do Estado do Amazonas, a 700 km de Manaus-AM e a 200 km de Porto Velho-RO, com coordenadas geográficas $7^{\circ}$ 32' 60”' (S) e $63^{\circ} 04^{\prime} 48^{\prime \prime}(\mathrm{O})$. A justificativa para essa localização é devida ao fato de essa região ter sido apontada como um dos locais no país onde muitos brasileiros vivem sem acesso ao serviço de energia elétrica, sendo assim caracterizada como um sistema isolado e desconectado do Sistema Interligado Nacional (SIN), o qual é coordenado pelo Operador Nacional do Sistema Elétrico (ONS) [11]. Essa localidade apresenta, ainda, peculiaridades por possuir uma superficie plana na maior parte das áreas, inclusive na direção crítica a ser estudada (os modelos de dispersão atmosférica se enquadram bem neste tipo de terreno), possuir área rural e urbana, lagos e rios, rodovias, além de ser próxima a estabelecimentos, do Exército Brasileiro e da Polícia Federal, o que pode favorecer ao aspecto da segurança física da instalação nuclear em estudo. Toda essa região e características geográficas podem ser visualizadas na Figura 4.

De acordo com a Figura 4, o reator está localizado a uma distância aproximada de: $250 \mathrm{~m}$ do Posto Fiscal da Sefaz/Polícia Rodoviária; $1 \mathrm{~km}$ do Instituto Federal de Educação, Ciência e Tecnologia; 1,2 km da Unidade do Exército Brasileiro; 1,5 km do Aeroporto Regional da localidade 
em estudo; 1,4 km do conjunto de residências assinalado nessa figura. Tais informações visam subsidiar a análise da dispersão atmosférica na fase inicial de resposta ao evento radiológico postulado, em que serão consideradas as ações protetoras imediatas, como a abrigagem e a evacuação, além da aplicação dos seus níveis genéricos de intervenção [12].

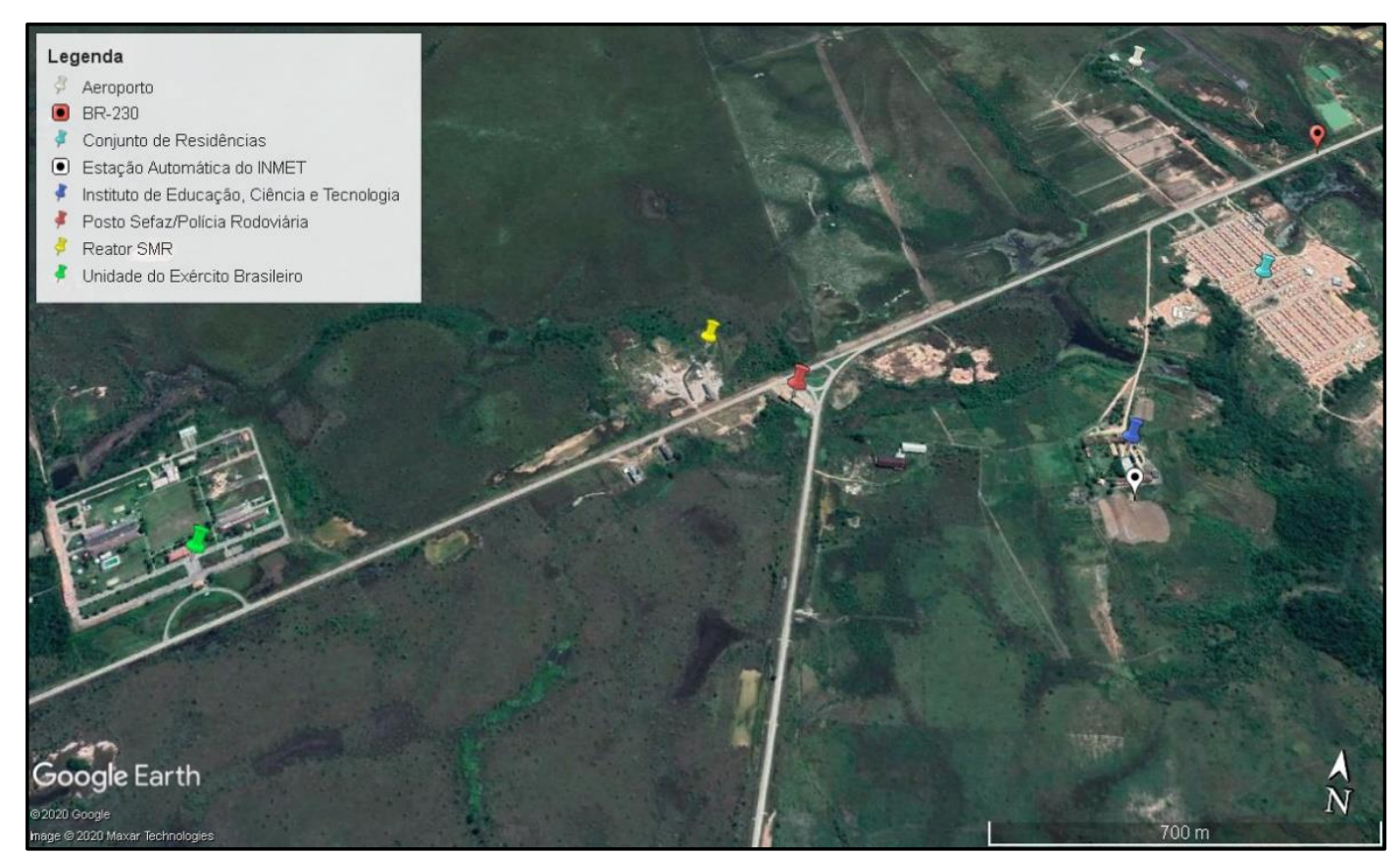

Figura 4: Visualização do local de instalação do SMR, mostrando áreas adjacentes.

Fonte: Google Earth, 2020

Para a simulação, foram coletadas informações sobre as condições climáticas e meteorológicas, a fim de se identificar as possíveis classes de estabilidade atmosférica existentes na região em estudo. Os dados foram obtidos de uma Estação Automática do Instituto Nacional de Meteorologia (INMET) da própria localidade, da base de dados do Instituto Nacional de Pesquisas Espaciais (INPE) e da literatura, considerando um período de observação relativo aos anos de 2014 a 2020.

No que se refere ao clima dessa região, ao longo do ano, em geral, a temperatura varia de $20^{\circ} \mathrm{C}$ a $32^{\circ} \mathrm{C}$ e raramente é inferior a $19^{\circ} \mathrm{C}$ ou superior a $35^{\circ} \mathrm{C}$. O período de chuvas é bem distribuído ao longo do ano, sendo mais representativo nos meses de janeiro a abril. Além disso, a umidade relativa do ar nessa cidade não varia significativamente ao longo do ano, permanecendo, em média, em $80 \%$, durante o ano inteiro [13-15]. 
Baseado nas informações meteorológicas, foram definidas a velocidade e a direção do vento na localidade em questão, durante todo o ano. Verificou-se que a velocidade do vento na região oscila no intervalo de 0 e $4 \mathrm{~m} / \mathrm{s}$, sendo que a maior velocidade registrada (rajadas) foi de $6 \mathrm{~m} / \mathrm{s}$. O período do dia em que essa velocidade máxima pode ocorrer está entre as $12 \mathrm{~h}$ até as $18 \mathrm{~h}$. A direção do vento é mais frequente entre $0^{\circ}$ a $90^{\circ}$, embora outras direções possam ocorrer ao longo do ano [13]. Além disso, a média obtida para intensidade da radiação solar, com os dados referentes ao período considerado, foi superior a $700 \mathrm{~W} / \mathrm{m}^{2}$ [14-16].

Com base nos dados climáticos e meteorológicos dessa localidade e admitindo-se um cenário plausível, assumiu-se que, por ocasião do evento radiológico postulado, a velocidade do vento era de $3 \mathrm{~m} / \mathrm{s}$, a liberação dos radionuclídeos para a atmosfera ocorreu no período diurno, não havia precipitações e a radiação solar era de $700 \mathrm{~W} / \mathrm{m}^{2}$, o que permite enquadrar a estabilidade atmosférica, para o presente estudo, na Classe A (extremamente instável) de Pasquill-Gifford [4]. Além disso, considerando-se as áreas adjacentes ao local de operação do SMR, a análise da dispersão atmosférica dos radionuclídeos foi realizada tomando por base a direção do vento vindo do Oeste $\left(270^{\circ}\right)$, no momento do acidente hipotético, situação em que serão ocasionados os maiores efeitos negativos e prejuízos, devido à existência de pontos relevantes (centro universitário, posto da Sefaz/Polícia Rodoviária e residências) na região de incidência e de deslocamento dos ventos, conforme legenda da Figura 4, justificando sua utilização neste estudo.

Dentre os radionuclídeos do inventário do SMR, a modelagem da dispersão atmosférica e o cálculo da TEDE foram conduzidos tomando por base a contribuição do Cs-137. A escolha desse radionuclídeo é justificada pelo caráter inovador desta proposta, ligada à ideia de adição de capacidades, convergindo plataformas computacionais estanques em torno de um objetivo comum. Assim, a opção por um radionuclídeo de relevância na área de proteção radiológica [3], para aplicação neste trabalho, favorece a utilização da mesma metodologia para situações mais gerais como no próprio caso do inventário de radionuclídeos do reator em questão, na sua totalidade.

Esse isótopo apresenta, ainda, notória importância na análise das consequências em acidentes radiológicos, por ser extremamente tóxico, apresentar uma meia-vida de 30 anos e pela elevada solubilidade em meio aquoso [3]. Diversos registros na literatura apontam os principais riscos de exposição, interna e externa, a esse radioisótopo, o que pode ser constatado, por exemplo, nos relatos do acidente de Goiânia, em 1987 [17]. 
Assim, de posse do modelo de dispersão, do tipo de terreno, das características do reator em estudo e das informações meteorológicas, esses dados foram analisados e inseridos como parâmetros de entrada no software HotSpot. Todos os cálculos foram realizados considerando a classe de estabilidade A de Pasquill-Guifford. A Tabela 1 apresenta esses parâmetros para a simulação do acidente radiológico provocado por uma suposta falha no cladding do SMR.

Tabela 1: Parâmetros de entrada para a simulação no HotSpot.

\begin{tabular}{lc}
\hline \multicolumn{1}{c}{ Parâmetros } & Dados de entrada \\
\hline Material radioativo & Cs-137, meia-vida: 30 anos \\
Atividade & Calculada pelo SCALE \\
Velocidade do vento $(H=10 \mathrm{~m})$ & $3,0 \mathrm{~m} / \mathrm{s}$ \\
Direção do vento & $270^{\circ}$ Oeste \\
Coordenadas de distância & Distância a partir da linha central da pluma \\
Classe de estabilidade (terreno padrão) & A (extremamente instável) \\
Altura do receptor & $1,5 \mathrm{~m}$ \\
Tempo de amostragem & 10 min \\
Fatores de conversão de dose $[18]$ & Federal Guidance Report $\mathrm{n}^{\circ} 11$ \\
Tempo de exposição & ${ }^{6}$ \\
Altura de liberação efetiva $(H)$ & Início: $00: 00 \mathrm{~h} ;$ Duração: $24 \mathrm{~h}$ \\
\hline
\end{tabular}

Sob as condições de contorno definidas na Tabela 1, foram delimitadas as zonas de estudo em curvas de isodoses, de acordo com os valores de TEDE recebidos, em função da distância à origem da dispersão. Essas curvas são organizadas como interna, intermediária e externa, assumindo os limites de $50 \mathrm{mSv}, 10 \mathrm{mSv}$ e $1 \mathrm{mSv}$, respectivamente. Os dois primeiros valores se referem à dose evitada pelas ações de evacuação $(50 \mathrm{mSv})$ e de abrigagem $(10 \mathrm{mSv})$ [12] e o terceiro $(1 \mathrm{mSv})$, apenas como um referencial para a terceira curva, em alusão ao limite anual de dose para um

\footnotetext{
${ }^{5}$ Altura em que estão localizados os órgãos mais radiossensíveis do corpo humano [4].

${ }^{6}$ Tempo em que o material radioativo depositado no solo deverá contribuir para a exposição da população pela via de exposição externa à radiação ionizante. Neste estudo, considerou-se a contribuição relativa a um dia de exposição [4].
} 
indivíduo do público ( $1 \mathrm{mSv} / \mathrm{ano}$ ), prevista para uma situação de exposição normal, antes do acidente [19].

Os resultados obtidos, em termos da TEDE recebida e da concentração do Cs-137, em diferentes distâncias a favor do vento, decorrentes da dispersão atmosférica, foram analisados e comparados com os limiares normativos estipulados pelo órgão regulador para a radioproteção, de forma a verificar a influência das condições meteorológicas sobre a pluma de contaminação, a população potencialmente afetada durante esse evento radiológico e, também, as medidas de proteção a serem tomadas na situação de emergência, isto é, na fase inicial do acidente descrito neste estudo.

Com isso, considerando um cenário estático pós-acidente, espera-se que o tamanho da pluma de contaminação radioativa seja dependente das classes de estabilidade de Pasquill-Gifford e da atividade do Cs-137 [3].

\section{RESULTADOS E DISCUSSÃO}

Por meio do software SCALE, foram obtidos os radionuclídeos, suas respectivas massas e atividades médias, bem como as frações de liberação correspondentes, para deduzir a atividade liberada na atmosfera após o acidente [20]. Com base nos resultados desse código, a Tabela 2 apresenta os 17 radionuclídeos mais importantes acumulados no reator, baseada na radiotoxicidade e nos possíveis danos que possam causar à saúde humana.

Tabela 2: Inventário dos radionuclídeos no SMR após 2 anos de operação.

\begin{tabular}{lcccc}
\hline \multirow{2}{*}{ Radionuclídeo } & \multicolumn{4}{c}{ Dados referentes ao inventário dos radionuclídeos, gerado pelo SCALE } \\
\cline { 2 - 5 } & Massa(g) & $\begin{array}{c}\text { Atividade }(\mathbf{B q}) \\
\text { no Núcleo }\end{array}$ & $\begin{array}{c}\text { Fração } \\
\text { de Liberação }\end{array}$ & $\begin{array}{c}\text { Atividade (Bq) } \\
\text { liberada na atmosfera }\end{array}$ \\
\hline Ba-140 & $3,409 \mathrm{E}+01$ & $9,228 \mathrm{E}+16$ & 0,02 & $1,846 \mathrm{E}+15$ \\
$\mathrm{Cs}-137$ & $1,337 \mathrm{E}+03$ & $4,295 \mathrm{E}+15$ & 0,30 & $1,289 \mathrm{E}+15$ \\
$\mathrm{I}-131$ & $1,032 \mathrm{E}+01$ & $4,746 \mathrm{E}+16$ & 0,40 & $1,898 \mathrm{E}+16$ \\
$\mathrm{I}-135$ & $7,453 \mathrm{E}-01$ & $9,744 \mathrm{E}+16$ & 0,40 & $3,898 \mathrm{E}+16$ \\
$\mathrm{Kr}-85$ & $4,252 \mathrm{E}+01$ & $6,153 \mathrm{E}+14$ & 1,00 & $6,153 \mathrm{E}+14$ \\
$\mathrm{Kr}-85 \mathrm{~m}$ & $5,700 \mathrm{E}-02$ & $1,735 \mathrm{E}+16$ & 1,00 & $1,735 \mathrm{E}+16$ \\
$\mathrm{Rn}-218$ & $5,137 \mathrm{E}-19$ & $2,811 \mathrm{E}+04$ & 1,00 & $2,811 \mathrm{E}+04$ \\
$\mathrm{Rn}-219$ & $1,613 \mathrm{E}-17$ & $7,766 \mathrm{E}+03$ & 1,00 & $7,766 \mathrm{E}+03$
\end{tabular}




\begin{tabular}{lllll} 
Rn-220 & $6,839 \mathrm{E}-13$ & $2,334 \mathrm{E}+07$ & 1,00 & $2,334 \mathrm{E}+07$ \\
Rn-222 & $1,327 \mathrm{E}-14$ & $7,553 \mathrm{E}+01$ & 0,02 & $1,511 \mathrm{E}+00$ \\
Sr-89 & $5,757 \mathrm{E}+01$ & $6,185 \mathrm{E}+16$ & 0,02 & $1,237 \mathrm{E}+15$ \\
Sr-90 & $7,325 \mathrm{E}+02$ & $3,739 \mathrm{E}+15$ & 0,02 & $7,479 \mathrm{E}+13$ \\
Te-132 & $6,049 \mathrm{E}+00$ & $6,911 \mathrm{E}+16$ & 0,05 & $3,455 \mathrm{E}+15$ \\
Xe-133 & $1,443 \mathrm{E}+01$ & $1,000 \mathrm{E}+17$ & 1,00 & $1,000 \mathrm{E}+17$ \\
Xe-133m & $7,293 \mathrm{E}-02$ & $1,210 \mathrm{E}+15$ & 1,00 & $1,210 \mathrm{E}+15$ \\
Xe-135 & $4,998 \mathrm{E}-01$ & $4,697 \mathrm{E}+16$ & 1,00 & $4,697 \mathrm{E}+16$ \\
Xe-135m & $4,102 \mathrm{E}-03$ & $1,383 \mathrm{E}+16$ & 1,00 & $1,383 \mathrm{E}+16$ \\
\hline
\end{tabular}

Assim, após os dados das Tabelas 1 e 2 (referentes ao Cs-137) serem inseridos no HotSpot, os valores de TEDE e o perfil de concentração são apresentados na Tabela 3, considerando-se uma distância máxima de $100 \mathrm{~km}$ da origem da dispersão. Os dados dessa tabela se referem ao período de um dia (24 horas) de exposição.

Tabela 3: Dados da dispersão do Cs-137 proveniente do acidente no SMR.

\begin{tabular}{lccc}
\hline & \multicolumn{3}{c}{ Dados referentes ao Cs-137 } \\
\cline { 2 - 4 } Distância $(\mathbf{k m})$ & TEDE $(\mathbf{S v})$ & Concentração no ar $\left[(\mathbf{B q . s}) / \mathbf{m}^{3}\right]$ & $\begin{array}{c}\text { Tempo de chegada da pluma } \\
\text { (hora:min) }\end{array}$ \\
\hline 0,03 & $3,50 \mathrm{E}+00$ & $1,80 \mathrm{E}+11$ & $<00: 01$ \\
0,1 & $9,90 \mathrm{E}-01$ & $5,50 \mathrm{E}+10$ & $<00: 01$ \\
0,5 & $4,00 \mathrm{E}-02$ & $2,50 \mathrm{E}+09$ & $00: 02$ \\
1 & $9,70 \mathrm{E}-03$ & $6,40 \mathrm{E}+08$ & $00: 05$ \\
2 & $2,40 \mathrm{E}-03$ & $1,70 \mathrm{E}+08$ & $00: 11$ \\
3 & $1,10 \mathrm{E}-03$ & $7,70 \mathrm{E}+07$ & $00: 16$ \\
4 & $6,10 \mathrm{E}-04$ & $4,50 \mathrm{E}+07$ & $00: 22$ \\
5 & $3,90 \mathrm{E}-04$ & $3,00 \mathrm{E}+07$ & $00: 27$ \\
10 & $1,10 \mathrm{E}-04$ & $8,60 \mathrm{E}+06$ & $00: 55$ \\
15 & $5,10 \mathrm{E}-05$ & $4,30 \mathrm{E}+06$ & $01: 23$ \\
20 & $3,10 \mathrm{E}-05$ & $2,60 \mathrm{E}+06$ & $01: 51$ \\
30 & $1,50 \mathrm{E}-05$ & $1,30 \mathrm{E}+06$ & $02: 46$ \\
40 & $9,50 \mathrm{E}-06$ & $8,50 \mathrm{E}+05$ & $03: 42$ \\
50 & $6,60 \mathrm{E}-06$ & $5,90 \mathrm{E}+05$ & $04: 37$ \\
60 & $4,90 \mathrm{E}-06$ & $4,40 \mathrm{E}+05$ & $05: 33$ \\
70 & $3,80 \mathrm{E}-06$ & $3,50 \mathrm{E}+05$ & $06: 28$ \\
80 & $3,10 \mathrm{E}-06$ & $2,80 \mathrm{E}+05$ & $07: 24$ \\
90 & $2,60 \mathrm{E}-06$ & $2,40 \mathrm{E}+05$ & $08: 20$ \\
100 & $2,20 \mathrm{E}-06$ & $2,00 \mathrm{E}+05$ & $09: 15$ \\
\hline
\end{tabular}


Da mesma forma, as Figuras 5 e 6 mostram as curvas de isodoses, representando ${ }^{7}$ a TEDE de 50 $\mathrm{mSv}, 10 \mathrm{mSv}$ e $1 \mathrm{mSv}$, respectivamente, geradas sobre o terreno, mostrando a área afetada, em que se considerou a classe de estabilidade extremamente instável (Classe A) atuando na região de operação do SMR.

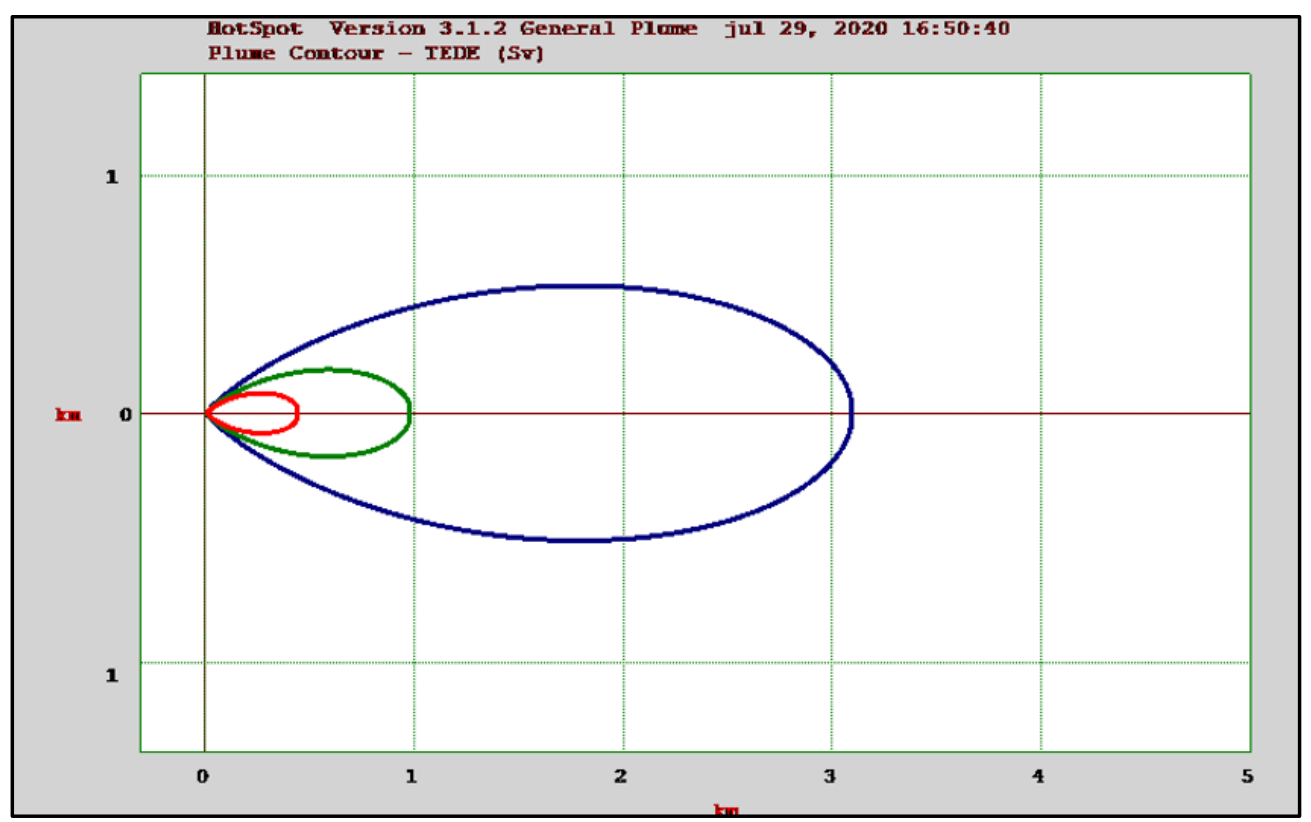

Figura 5: Curvas de isodose devido ao Cs-137, obtidas no HotSpot, considerando a classe de estabilidade A. Isodose interna (cor vermelha): $50 \mathrm{mSv}\left(0,055 \mathrm{~km}^{2}\right)$; Isodose intermediária (cor verde): $10 \mathrm{mSv}\left(0,26 \mathrm{~km}^{2}\right)$; Isodose externa (cor azul): $1 \mathrm{mSv}\left(2,4 \mathrm{~km}^{2}\right)$.

Fonte: HOMANN, 2019

\footnotetext{
${ }^{7}$ A data e a hora previstas nas Figuras 5 e 7 deste trabalho se referem ao período da simulação no HotSpot e não estão relacionadas ao horário (data e hora) da dispersão do Cs-137 para a atmosfera.
} 


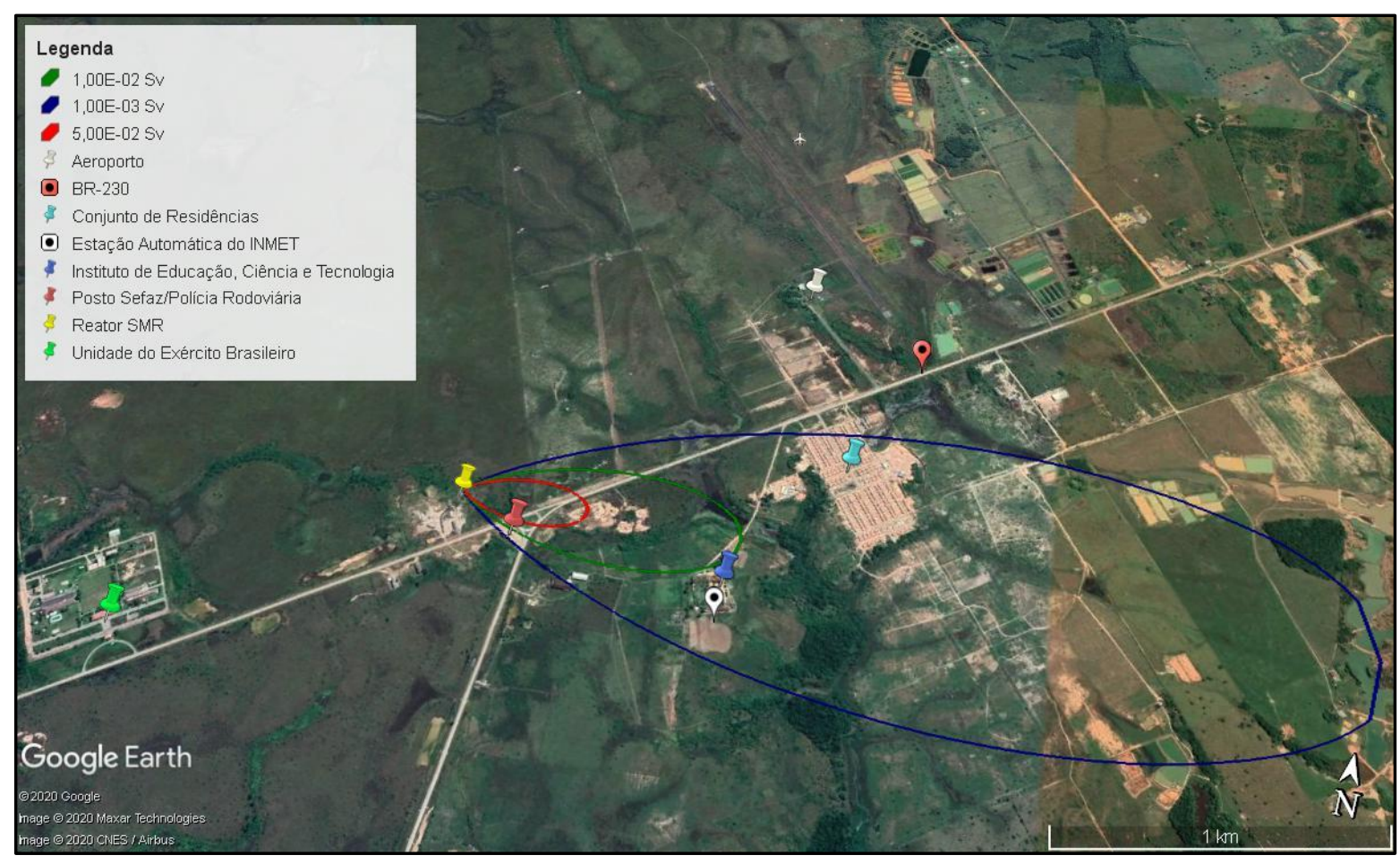

Figura 6: Limite de contorno das isodoses referentes à pluma de contaminação com Cs-137. Fonte: Google Earth, 2020

A tabela 4 apresenta outras informações importantes para análise da dispersão atmosférica, considerando-se as premissas adotadas na simulação.

Tabela 4: Dados obtidos da simulação computacional no HotSpot referentes à dispersão do Cs-137.

\begin{tabular}{lc}
\hline \multicolumn{1}{c}{ Informação } & Observação \\
\hline Valor Máximo de TEDE & $3,6 \mathrm{~Sv}$ \\
Distância do SMR em que a TEDE é máxima & $0,034 \mathrm{~km}$ \\
Distância do SMR em que a TEDE excede a isodose interna & $0,45 \mathrm{~km}$ \\
Distância do SMR em que a TEDE excede a isodose intermediária & $0,98 \mathrm{~km}$ \\
Distância do SMR em que a TEDE excede a isodose externa & $3,1 \mathrm{~km}$ \\
\hline
\end{tabular}

Foi realizado também o cálculo do transporte atmosférico do Cs-137 para comparação com outras classes de estabilidade, na hipótese de existir na região em estudo, diferentes classes atmosféricas atuando na localidade em que se encontra operando o reator, relacionando-se os 
valores de dose recebida em diferentes distâncias a partir da origem da dispersão desse radionuclídeo, bem como o valor máximo de TEDE alcançado, nas diferentes classes de estabilidade, conforme descrito na Figura 7 e apresentado na Tabela 5.

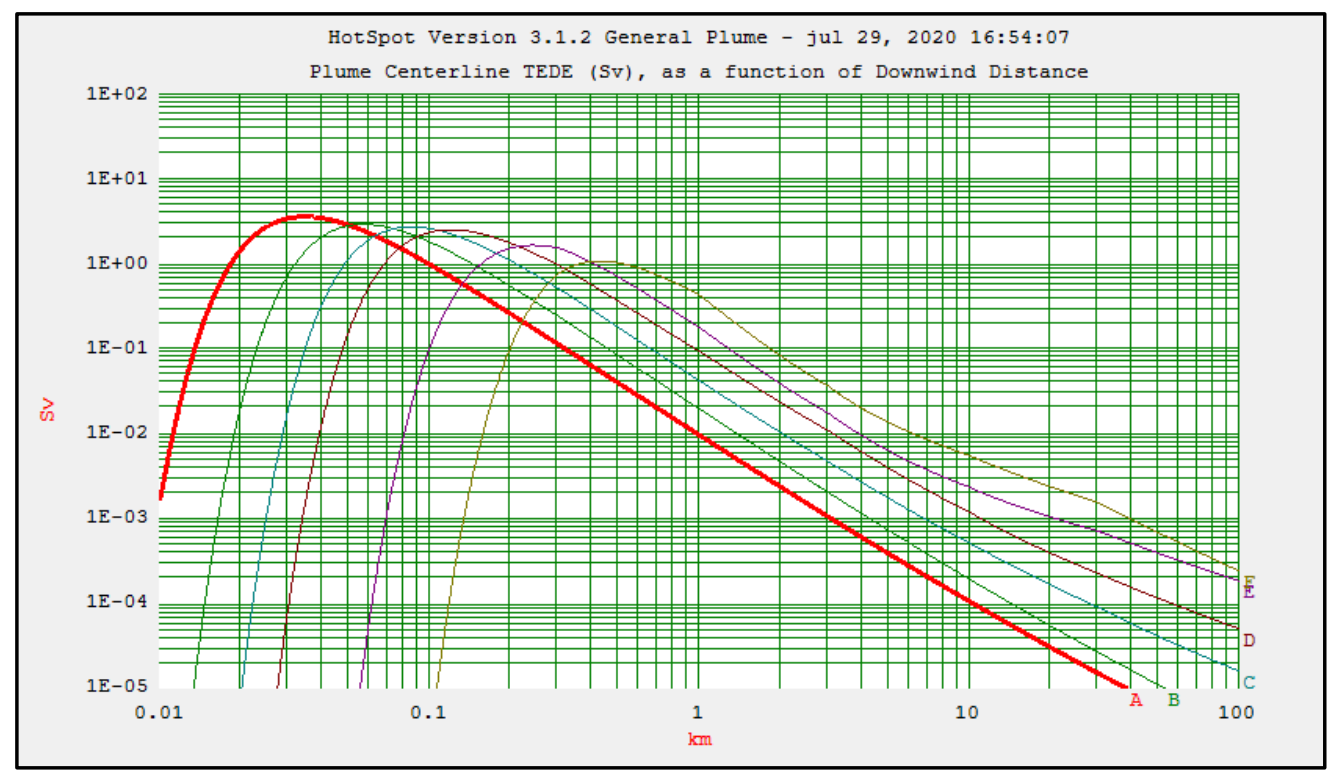

Figura 7: Valores de TEDE recebida em diferentes distâncias a favor do vento, considerando todas as classes de estabilidade.

Fonte: HOMANN, 2019

Tabela 5: Valores máximos de TEDE para diferentes classes de estabilidade.

\begin{tabular}{cccc}
\hline \multirow{2}{*}{$\begin{array}{c}\text { Classe de } \\
\text { Estabilidade }\end{array}$} & \multicolumn{2}{c}{ Dados referentes à dispersão do Cs-137 } \\
\cline { 2 - 4 } Atmosférica & Descrição & $\begin{array}{c}\text { TEDE } \\
\text { máxima (Sv) }\end{array}$ & Distância (km) do SMR \\
\hline A & Extremamente instável & 3,6 & 0,034 \\
B & Moderadamente instável & 2,9 & 0,057 \\
C & Levemente instável & 2,8 & 0,085 \\
D & Condições neutras & 2,5 & 0,120 \\
E & Levemente estável & 1,7 & 0,240 \\
F & Moderadamente estável & 1,1 & 0,430 \\
\hline
\end{tabular}

A partir da origem da dispersão, os valores calculados mostraram que a máxima TEDE, devido ao Cs-137, é obtida a 34 m, com um valor de 3,6 Sv, diminuindo com o tempo e a distância. Esses resultados são obtidos em momentos diferentes, à medida que esse material radioativo atinge pontos receptores designados em locais a favor do vento [3], conforme mostrado na Tabela 3. Tal 
comportamento se demonstra aceitável do ponto de vista teórico, uma vez que a liberação desse radionuclídeo para a atmosfera segue o modelo Gaussiano de dispersão, apresentado na Figura 3 $[4,9]$.

Pelas Figuras 5 e 6, verifica-se que os valores mais altos de dose, acima de $50 \mathrm{mSv}$, são encontrados na área entre o ponto zero e $0,45 \mathrm{~km}$, seguido pelo valor intermediário de dose, maiores do que $10 \mathrm{mSv}$ e menores do que $50 \mathrm{mSv}$, dentro da distância de 0,45 km a 0,98 km, e a última curva na distância de $0,98 \mathrm{~km}$ a 3,1 km, representando os valores acima de $1 \mathrm{mSv}$ e menores do que $10 \mathrm{mSv}$. As curvas abrangem uma área de $0,055 \mathrm{~km}^{2}, 0,26 \mathrm{~km}^{2}$ e 2,4 km², respectivamente.

Com relação à influência das condições meteorológicas na dispersão atmosférica, os resultados demonstraram que, quanto mais instáveis essas condições prevalecem no local, maior o valor da TEDE a uma menor distância a partir da origem da dispersão do Cs-137, conforme apresentado na Tabela 5. Isso pode ser comprovado na Figura 7, que mostra a diminuição nos valores máximos de TEDE quando as condições de estabilidade atmosférica se tornam mais estáveis. Além disso, pelas curvas apresentadas nessa mesma figura, verifica-se, também, um aumento na TEDE para distâncias maiores quando se considera as classes mais estáveis. Essas observações sugerem que as situações de elevada turbulência na atmosfera favorecem a mistura de poluentes, aumentando a taxa de deposição do material radioativo no solo e diminuindo o transporte desse radionuclídeo para maiores distâncias, isto é, gerando menores valores de dose proveniente da submersão à nuvem de Cs-137. Além disso, os resultados também denotam a depedência da pluma de contaminação radioativa com os critérios de estabilidade atmosférica, propostos por Pasquill-Gifford [3,4].

Com relação às medidas de proteção em situações de emergência, constatada a ocorrência radiológica pelo serviço de emergência da instalação, uma rápida avaliação deve ser realizada pela equipe de campo, com ênfase particular nas áreas densamente povoadas, de modo que as técnicas de monitoração forneçam dados tanto mais próximos da realidade quanto possível, a fim de auxiliar a coordenação da emergência a adotar as ações ditas protetoras.

Assim sendo, de acordo com os dados calculados, para doses a partir de $50 \mathrm{mSv}$, a intervenção poderá ocorrer visando à evacuação do pessoal das proximidades do reator, em movimento contrário ao de propagação da pluma. Conforme características geográficas apresentadas na Figura 4, e de acordo com o cenário de dispersão atmosférica assumido (período diurno, direção principal do vento vindo do Oeste e sem chuvas no momento do acidente postulado), existem locais nessa 
região, como o Aeroporto e também a Unidade do Exército Brasileiro, que além de estarem próximos ao local do reator, não estão sendo influenciados pelo deslocamento preferencial da pluma, o que permitiria a execução dessa ação protetora. Além disso, a existência da BR-230 facilitaria o transporte e a remoção do pessoal para os locais assinalados, desde que essa medida ocorra por um período de até 1 (uma) semana, conforme recomendado pelas autoridades regulatórias [12].

Para as doses compreendidas no intervalo entre $10 \mathrm{mSv}$ e abaixo de $50 \mathrm{mSv}$, os resultados sugerem a adoção da abrigagem como medida protetora. Conforme verificado nas curvas de isodoses da Figura 6, o Posto Fiscal da Sefaz/Polícia Rodoviária, o Instituto Federal de Educação, Ciência e Tecnologia e o conjunto de residências existentes estão recebendo exposições inferiores a $10 \mathrm{mSv}$. Esse mesmo procedimento, relativo à abrigagem, também pode ser adotado para a população que estiver submetida às doses compreendidas no intervalo entre $1 \mathrm{mSv}$ e abaixo de 10 mSv, uma vez que em situações de emergência, os níveis de intervenção pré-estabelecidos podem ser reavaliados, quando de sua implementação, em função das condições existentes no momento do acidente, desde que não sejam excedidos os níveis de dose (no caso da abrigagem, representados no intervalo entre $10 \mathrm{mSv}$ e abaixo de $50 \mathrm{mSv}$ ) [12]. Essa medida reduziria consideravelmente as doses relativas à irradiação proveniente da nuvem de Cs-137, à irradiação oriunda do solo contaminado e à inalação desse material radioativo, devido ao fator de redução inerente a essas construções.

Entretanto, mesmo no caso das doses a partir de $50 \mathrm{mSv}$, a abrigagem poderá ser adotada como medida protetora, considerando os abrigos descritos no parágrafo anterior. O momento ideal para implementar a evacuação seria antes da passagem da pluma, o que pode não ser possível diante dos resultados alcançados neste estudo, uma vez que a nuvem radioativa de Cs-137 alcança os pontos mais próximos do reator em menos de 5 min. Caso a evacuação fosse executada durante a passagem da pluma, seria possível que doses mais elevadas fossem recebidas pelos evacuandos do que por aqueles que permanecerem abrigados. Além disso, dependendo das condições meteorológicas, da quantidade de pessoas a serem evacuadas e dos meios de transporte disponíveis, a evacuação poderia requerer muito tempo, corroborando a opção pelos abrigos, de forma a não colocar em risco a população potencialmente envolvida no acidente. Cabe ressaltar que a abrigagem não deve ser aplicada por um período superior a dois dias [12]; caso seja previsto que estas doses possam ocorrer 
por um período maior, a evacuação, em uma etapa posterior, isto é, após a passagem da pluma, poderia ser mais efetiva.

A partir da distância de 3,1 km da origem da dispersão do Cs-137, os valores de TEDE, em diferentes pontos, estão abaixo de $1 \mathrm{mSv}$. Os resultados sugerem que esse radionuclídeo pode ser transportado para longas distâncias, entretanto, os valores de dose devido à contribuição desse material radioativo, na quantidade em que foi liberada para atmosfera, conforme os dados da Tabela 1, estarão abaixo dos níveis de intervenção propostos para a abrigagem e a evacuação, não sendo, portanto, necessária a adoção dessas medidas protetoras, nas condições simuladas [3]. Esses valores, inclusive, estão na mesma ordem de grandeza daqueles permitidos para situações de operação normal, antes do momento do acidente, em que o limite de dose é de $1 \mathrm{mSv} / \mathrm{ano}$ para o indívíduo do público [19].

\section{CONCLUSÕES}

O objetivo da pesquisa foi alcançado por meio da simulação por modelagem computacional da dispersão atmosférica decorrente de um acidente radiológico hipotético em um reator modular de pequeno porte SMR (50 MWt), produtor de energia elétrica, em uma região onde o seu abastecimento não é pelo Operador Nacional, para analisar a adoção das medidas protetoras imediatas na fase inicial do evento radiológico postulado, bem como a influência das condições meteorológicas na pluma de contaminação.

Os principais resultados desta pesquisa mostraram que, para a classe de estabilidade A, assumida no momento do acidente hipotético, um valor máximo de TEDE, de 3,6 Sv, foi obtido, a uma distância de $34 \mathrm{~m}$ da origem da dispersão, como contribuição de um radionuclídeo com importância significativa na proteção radiológica, o Cs-137, o qual foi obtido do inventário do SMR, cujo cálculo foi realizado na condição de burnup total desse reator. A partir desse resultado, o presente estudo permitiu verificar que os demais valores de concentração e de dose calculados, referentes a esse radionuclídeo, diminuem com o tempo e a distância, seguindo o modelo Gaussiano de dispersão [4,9].

A pesquisa também permitiu verificar a influência das condições meteorológicas na dispersão atmosférica, mostrando a depedência da pluma de contaminação radioativa com os critérios 
propostos por Pasquill-Gifford. Os resultados demonstraram que quanto mais instáveis essa condições prevalecem no local, maior o valor da TEDE a uma menor distância a partir da origem da dispersão do Cs-137, e que ocorre um aumento nas doses, para distâncias maiores, quando se consideram as classes de estabilidade atmosférica mais estáveis.

Outro achado relevante, fruto deste estudo, é que, considerando apenas a liberação de Cs-137, para doses a partir de $50 \mathrm{mSv}$, a intervenção poderá ocorrer no sentido da evacucação do pessoal das proximidades do reator, em um período inferior a uma semana, tanto para Aeroporto da região, como também para a Unidade do Exército Brasileiro, caracterizando um movimento contrário à direção preferencial da pluma. Para as doses compreendidas no intervalo entre $10 \mathrm{mSv}$ e abaixo de $50 \mathrm{mSv}$ e entre $1 \mathrm{mSv}$ e abaixo de $10 \mathrm{mSv}$, o resultados sugerem a adoção da abrigagem como medida protetora, considerando que na localidade do reator existem o Posto Fiscal da Sefaz/Polícia Rodoviária, o Instituto Federal de Educação, Ciência e Tecnologia e as residências, que recebem exposição inferiores a $10 \mathrm{mSv}$. Além disso, para todos esses casos, dependendo do tempo de chegada da pluma, das condições meteorológicas, da quantidade de pessoal a ser removido e dos meios de transporte existentes, a opção pelos abrigos pode ser a mais relevante, desde que não ultrapasse os dois dias de abrigagem, momento a partir do qual a evacuação é a opção mais eficaz.

Sendo assim, a pesquisa mostra que, para estudos de dispersão atmosférica, com a caracterização bem definida da fonte de radiação ionizante, a metodologia aplicada na modelagem computacional de um acidente radiológico em um SMR permite verificar o atendimento das normas quanto ao planejamento de respostas em situação de emergência e à influência das condições meteorológicas.

\section{AGRADECIMENTOS}

A equipe agradece ao Professor Sérgio de Oliveira Vellozo pelo suporte fornecido no uso do código computacional SCALE. Também ao Instituto Militar de Engenharia (IME) e ao Instituto de Defesa Química Biológica Radiológica e Nuclear (IDQBRN) pelo apoio dado para o desenvolvimento deste trabalho. Por fim, à Coordenação de Aperfeiçoamento de Pessoal de Nível 
Superior (CAPES) e ao Conselho Nacional de Desenvolvimento Científico e Tecnológico (CNPq) por todo auxílio financeiro.

\section{REFERÊNCIAS}

[1] FISCHER, G., RIPLEY, C. Improving Air-Cooled Condenser Performance and Availability Through Innovative Cleaning, Inspection and In-Situ Sleeving. In: 2012 20th International Conference on Nuclear Engineering and the ASME 2012 Power Conference. American Society of Mechanical Engineers Digital Collection, 2012. p. 777-782.

[2] UNITED STATES DEPARTMENT OF ENERGY - DOE (2019). Reactor Technology: Benefits of Small Modular Reactors (SMRs). Disponível em: <https://www.energy.gov/ne/benefits-small-modular-reactors-smrs>. Acesso em: 10/02/2020.

[3] MUSWEMA, J. L. et al. Atmospheric dispersion modeling and radiological safety analysis for a hypothetical accident of Ghana Research Reactor-1 (GHARR-1). Annals of Nuclear Energy, v. 68, p. 239-246, 2014.

[4] HOMANN, S. G. Hotspot Health Physics Codes Version 3.1 User's Guide. CA, USA: Lawrence Livermore National Laboratory, 2019.

[5] REARDEN, B. T.; JESSEE, M. A. SCALE code system, ORNL/TM-2005/39, version 6.2.1. Oak Ridge National Laboratory, Oak Ridge, Tennessee, 2016.

[6] NETO A. T., DUARTE, G.M., TALON, J.D., LOPES, T.J., OLIVEIRA, C.L., FIEL, J.C.B., FONTES, G.S., CABRAL, R.G., VEllozO, S.O., BARROSO, D.E.G. Blindagem multilaminada aplicada a um reator modular de pequeno porte hipotético. BJRS, 2019.

[7] DUDERSTADT, James J. Nuclear reactor analysis. Wiley, 1976.

[8] SMITH, Matthew C.; WRIGHT, Richard F. Westinghouse small modular reactor passive safety system response to postulated events. Proc. of ICAPP'12, 2012.

[9] STOCKIE, John M. The mathematics of atmospheric dispersion modeling. Siam Review, v. 53, n. 2, p. 349-372, 2011.

[10] US.NRC. Basics References, Glossary. Disponível em: <https://www.nrc.gov/readingrm/basic-ref/glossary/total-effective-dose-equivalent-tede.html >. Acesso em: 03/03/2020. 
[11] IEMA. Matriz elétrica limpa e inclusiva. Disponível em:

$<$ http://energiaeambiente.org.br/um-milhao-estao-sem-energia-eletrica-na-amazonia20191125>. Acesso em: 05/03/2020.

[12] COMISSÃO NACIONAL DE ENERGIA NUCLEAR (CNEN). Posição regulatória 3.01/006. Medidas de Proteção e Critérios de Intervenção em Situações de Emergência. Rio de Janeiro, 2011.

[13] SIQUEIRA, Luan Ferreira et al. Caracterização do regime de ventos nos municipios de Humaitá-Am e Apuí-Am. EDUCAmazônia, v. 12, n. 1, p. 99-112, 2014.

[14] INPE. Instituto Nacional de Pesquisas Espaciais. Disponível em:

<http://clima1.cptec.inpe.br/monitoramentobrasil/pt>. Acesso em: 25/07/2020.

[15] INMET. Instituto Nacional de Meteorologia. Disponível em:

<https://portal.inmet.gov.br/dadoshistoricos>. Acesso em: 25/07/2020.

[16] Condições meteorológicas médias de Humaitá. Weather Spark, 2020. Disponível em:

<https://pt.weatherspark.com/y/28384/Clima-caracter\%C3\%ADstico-em-Humait\%C3\%A1-

Brasil-durante-o-

ano\#: :text=Em\%20Humait\%C3\%A1\%2C\%20a\%20esta\%C3\%A7\%C3\%A3o\%20com,superio r\%20a\%2036\%20\%C2\%B0C.>. Acesso em: 25/07/2020.

[17] INTERNATIONAL ATOMIC ENERGY AGENCY (1988), The Radiological Accident in Goiânia. IAEA, Vienna.

[18] Eckerman, K. F.; Wolbarst, A. B.; Richardson, A. C. B. Federal Guidance Report No. 11:

Limiting Values of Radionuclide Intake and Air Concentration and Dose Conversion Factors for Inhalation, Submersion, and Ingestion. EPA, 1988.

[19] COMISSÃO NACIONAL DE ENERGIA NUCLEAR (CNEN). Diretrizes Básicas de Proteção Radiológica. Norma CNEN NN 3.01. Rio de Janeiro, 2014.

[20] US NUCLEAR REGULATORY COMMISSION et al. Regulatory Guide 1.183 (draft wasIssuedas DG-1081): Alternative Radiological Source Terms for Evaluating Design Basis Accidents at Nuclear Power Reactors. US Nuclear Regulatory Commission, Office of Nuclear Regulatory Research, 2000. 